\title{
On a set-valued functional integral equation of Volterra- Stieltjes type
}

\author{
A. M. A. El-Sayed ${ }^{a}$, Sh. M Al-Issab,c,* \\ ${ }^{a}$ Faculty of Science, Alexandria University, Alexandria, Egypt. \\ ${ }^{b}$ Faculty of Science, Lebanes International University, Beirut, Lebanon. \\ ${ }^{c}$ Faculty of Science, The International University of Beirut, Saida, Lebanon.
}

\begin{abstract}
In this paper, we study the existence of continuous solutions of a set-valued functional integral equation for the VolterraStieltjes type. The asymptotic stability of the solutions will be studied. The continuous dependence of the solution on the set of selections of the set-valued function will be proven. As an application, we study the existence of solutions of an initial value problem of arbitrary (fractional) order differential inclusion.
\end{abstract}

Keywords: Nonlinear Volterra-Stieltjes integral inclusion, function of bounded variation, asymptotic stability, continuous dependence of the solution, integral inclusion of fractional order.

2020 MSC: 74H10, 45G10.

(C)2020 All rights reserved.

\section{Introduction}

The topic of fractional calculus is enjoying growing interest not only among mathematicians but also among physicists and engineers (see $[6,10,19,26,29,30])$. It turns out that a lot of results of the theory of differential and integral equations of fractional order can be considered from a unified point of view with help of the theory of the so-called Volterra-Stieltjes set-valued integral equation. A set-valued functional equation has been extensively investigated by a number of authors and there are many interesting results concerning this problem (see [15-18, 20, 23, 24, 27]). The interest in the study of Volterra-Stieltjes integral equations was initiated mainly by the papers (see $[4,12,13,21]$ ).

In this paper, we discuss the existence of continuous solutions for the set-value functional integral equation of Volterra-Stiltjes type

$$
x(t) \in p(t)+F_{1}\left(t, \int_{0}^{t} f_{2}\left(s, x(\varphi(s)) d_{s} g(t, s)\right), \quad t \in[0, T], T<\infty,\right.
$$

where $F_{1}:[0, T] \times R^{+} \rightarrow P(R)$ is a set-valued mapping and $P(R)$ denotes the family of nonempty subsets of $R$. Our study depends on the selections of the set-valued function $F_{1}$ by reformulating the Volterra-Stiltjes

\footnotetext{
${ }^{*}$ Corresponding author

Email address: shorouk. alissa@liu.edu.lb (Sh. M Al-Issa)

doi: $10.22436 /$ jmcs.021.04.01

Received: 2020-03-08 Revised: 2020-03-15 Accepted: 2020-04-08
} 
integral inclusion (1.1) into a coupled system. The asymptotic stability of the solutions will be studied. Also, the continuous dependence of the solution on the set of selections of the set-valued function $F_{1}$ and function $g(t, s)$ will be proved. As particular cases, the existence theorems concerning both nonlinear integral inclusion of fractional orders

$$
x(t) \in p(t)+F_{1}\left(t, I^{\alpha} f_{2}(t, x(\varphi(t)))\right), \quad t \in[0, T], \quad T<\infty
$$

and the initial value problem of arbitrary (fractional) orders differential inclusion

$$
\begin{aligned}
& \frac{d x(t)}{d t} \in F_{1}\left(t, D^{\beta} x(t)\right), \quad t \in(0, T], \quad T<\infty, \\
& x(0)=x_{0},
\end{aligned}
$$

were studied where $\alpha, \beta \in(0,1]$, and some further results will be discussed. Indeed, the tools and methods associated with the theory of nonlinear Volterra-Stieltjes set-valued integral equations which will be applied in this paper are more convenient and allow us to obtain more applicable results.

This paper is organized as follows. In Section 2, we recall some useful preliminaries. In Section 3, we state sufficient conditions which guarantee the existence of at least one continuous solution to the problem (1.1). In Section 4, we show that the solutions of the nonlinear Volterra-Stieltjes integral inclusion are asymptotically stable. While Section 5, deals with the existence of continuous dependence of unique solutions for inclusion (1.1) on the set of selections of the set-valued function and on the function $g(t, s)$. Also, we deduce the existence theorems of solutions for Volterra integral equation of fractional order and differential inclusion in Section 6. In Section 7, we discuss some special cases of inclusion (1.1).

\section{Preliminaries}

This section is devoted to providing the notation, definitions, and other auxiliary facts that will be needed in our further study. At the beginning assume that $E$ is a Banach space with the norm $\|\cdot\|_{E}$. For an interval, $\mathrm{I}=[0, T]$, where $\mathrm{T}<\infty$, denote by $\mathrm{C}=\mathrm{C}(\mathrm{I}, \mathrm{E})$ the space consisting of all continuous functions defined on I and taking values in the space $E$. This space will be furnished with sup-norm

$$
\|x\|_{C}=\sup _{t \in I}|x(t)| .
$$

We will accept the following axiomatic definition and theorem of the concept of a set-valued map.

Definition 2.1. Let $F$ be a set-valued map defined on a Banach space $E, f$ is called a selection of $F$ if $f(x) \in F(x)$, for every $x \in E$ and we denote by

$$
S_{F}=\{f: f(x) \in F(x), x \in E\}
$$

the set of all selections of $F$ (for the properties of the selection of $F$ see $[9,25,27]$ ).

Definition 2.2 ([23]). A set-valued map $F$ from $I \times E$ to family of all nonempty closed subsets of $E$ is called Lipschitzian if there exists $k>0$ such that for all $t \in I$ and all $x_{1}, x_{2} \in E$, we have

$$
h\left(F\left(t, x_{1}\right), F\left(s, x_{2}\right)\right) \leqslant k\left(|t-s|+\left|x_{1}-x_{2}\right|\right),
$$

where $h(A, B)$ is the Hausdorff distance between the two subsets $A, B \in I \times E$ (for the properties of the Hausdorff distance see [3]).

The following Theorem [3, Sect.9, Chap.1, Th.1] assumes the existence of Lipschitzian selection.

Theorem 2.3 ([24]). Let $M$ be a metric space and $\mathrm{F}$ be Lipschitzian set-valued function from $M$ into the nonempty compact convex subsets of $\mathrm{R}^{\mathrm{n}}$. Assume, moreover, that for some $\lambda>0, \mathrm{~F}(\mathrm{x}) \subset \lambda \mathrm{B}$ for all $\mathrm{x} \in \mathrm{M}$, where $\mathrm{B}$ is the unit ball on $R^{n}$. Then there exists a constant $c$ and a single-valued function $f: M \rightarrow R^{n}, f(x) \in F(x)$ for $x \in M$; this function is Lipschitzian with constant $\mathrm{k}$. 
In what follows, we discuss a few auxiliary facts concerning the functions of bounded variation [2]. To this end assume that $x$ is a real function defined on a fixed interval $[a, b]$. By the symbol $\bigvee_{a}^{b} x$ we will denote the variation of the function $x$ on the interval $[a, b]$. In the case when $\bigvee_{a}^{b} x$ is finite we say that $x$ is of bounded variation on $[a, b]$. In the case of a function $u(t, s)=:[a, b] \times[c, d] \rightarrow R$ we can consider the variation $\bigvee_{t=p}^{q} u(t, s)$ of the function $t \rightarrow u(t, s)$ (i.e., the variation of the function $u(t, s)$ with respect to the variable $t)$ on the interval $[p, q] \subset[a, b]$. Similarly, we define the quantity $\bigvee_{s=p}^{q} u(t, s)$. We will not discuss the properties of the variation of functions of bounded variation. We refer to [2] for the mentioned properties. Furthermore, assume that $x$ and $\phi$ are two real functions defined on the interval $[a, b]$. Then, under some extra conditions (cf. [2]), we can define the Stieltjes integral (more precisely, the RiemannStieltjes integral) of the function $x$ with respect to the function $\phi$ on the interval $[a, b]$ which is denoted by the symbol $\int_{a}^{b} x(t) d_{\phi}(t)$. In such a case, we say that $x$ is Stieltjes integrable on the interval $[a, b]$ with respect to $\phi$.

In the relevant literature, we may encounter a lot of conditions guaranteeing the Stieltjes integrability $[2,22,27]$. One of the most frequently exploited conditions requires that $x$ is continuous and $\phi$ is of bounded variation on $[a, b]$. [2]).

Next, we recall a few properties of the Stieltjes integral which will be used in our considerations (cf.

Lemma 2.4. Assume that $\mathrm{x}$ is Stieltjes integrable on the interval $[\mathrm{a}, \mathrm{b}]$ with respect to a function $\phi$ of bounded variation. Then

$$
\left|\int_{a}^{b} x(t) d_{\phi}(t)\right| \leqslant \int_{a}^{b}|x(t)| d\left(\bigvee_{a}^{t} \phi\right) .
$$

Lemma 2.5. Let $\mathrm{x}_{1}$ and $\mathrm{x}_{2}$ be Stieltjes integrable functions on the interval, $[\mathrm{a}, \mathrm{b}]$ with respect to a nondecreasing function $\phi$ such that $\mathrm{x}_{1}(\mathrm{t}) \leqslant \mathrm{x}_{2}(\mathrm{t})$ for $\mathrm{t} \in[\mathrm{a}, \mathrm{b}]$. Then the following inequality is satisfied:

$$
\int_{a}^{b} x_{1}(t) d_{\phi}(t) \leqslant \int_{a}^{b} x_{2}(t) d_{\phi}(t)
$$

In the sequel, we will also consider the Stieltjes integrals of the form $\int_{a}^{b} x(s) d_{s} g(t, s)$, where $g:[a, b] \times$ $[a, b] \rightarrow R$ and the symbol $d_{s}$ indicates the integration with respect to the variables. The details concerning the integral of such a type will be given later.

\section{Existence of at least one continuous solution}

Consider now the set-valued integral equation (1.1) under the following assumptions

(i) $p: I \rightarrow R$ is a continuous function on $I, p^{*}=\sup _{t \in I}|p(t)|$;

(ii) $F_{1}: I \times R \rightarrow P(R)$ is a Lipschitzian set-valued map with a nonempty compact convex subset of $2^{R^{+}}$;

(iii) $\varphi: I \rightarrow I$ is a continuous function such that $\varphi(t) \leqslant t$;

(iv) $f_{2}: I \times R \rightarrow R$ is continuous and there exist two constants $a$ and $b$ such that

$$
\left|f_{2}(t, x)\right| \leqslant a+b|x|, \quad \forall t \in I \text { and } x \in R ;
$$

(v) the function $g(t, s)=g: \Lambda \rightarrow R$ is continuous on $\Lambda=\{(t, s): 0 \leqslant s \leqslant t \leqslant T\}$;

(vi) the function $s \rightarrow g(t, s)$ is of bounded variation on $[0, t]$ for each $t \in I$;

(vii) for any $\epsilon>0$, there exists $\delta>0$ such that, for all $t_{1} ; t_{2} \in I$ with $t_{1}<t_{2}$ and $t_{2}-t_{1} \leqslant \delta$, the following inequality holds:

$$
\bigvee_{s=0}^{t_{1}}\left[g\left(t_{2}, s\right)-g\left(t_{1}, s\right)\right] \leqslant \epsilon ;
$$


(viii) $g(t, 0)=0$ for any $t \in I$.

It is clear that from Theorem 2.3 and assumption (ii), the set of Lipschitz selection of $F_{1}$ is non empty. So, the solution of the single-valued integral equation

$$
x(t)=p(t)+f_{1}\left(t, \int_{0}^{t} f_{2}\left(s, x(\varphi(s)) d_{s} g(t, s)\right), \quad t \in I,\right.
$$

where $f_{1} \in S_{F_{1}}$, is a solution to the inclusion (1.1). It must be noted that $f_{1}$ satisfied the Lipschitz selection

$$
\left|f_{1}(t, x)-f_{1}(s, y)\right| \leqslant k(|t-s|,|x-y|) .
$$

Obviously, we will assume that $g$ satisfies assumptions (v)-(viii).

For our purposes, we will only need the following lemmas.

Lemma 3.1 ([6]). The function $z \rightarrow \bigvee_{s=0}^{z} g(t, s)$ is continuous on $[0, t]$ for any any $t \in I$.

Lemma 3.2 ([6]). Let the assumptions (vi)-(vii) be satisfied. Then, For an arbitrary fixed number $0<\mathrm{t}_{2} \in \mathrm{I}$ and for any $\epsilon>0$, there exists $\delta>0$ such that if $t_{1} \in I ; t_{1}<t_{2}$ and $t_{2}-t_{1} \leqslant \delta$ then $\bigvee_{s=t_{1}}^{t_{2}} g\left(t_{2}, s\right) \leqslant \epsilon$.

Lemma 3.3 ([6]). Under the the assumptions (vi)-(vii), the function $t \rightarrow \bigvee_{s=0}^{t} g(t, s)$ is continuous on $\mathrm{I}$.

Further, let us observe that based on Lemma 3.3, we infer that there exists a finite positive constant K, such that

$$
K=\sup \left\{\bigvee_{s=0}^{t} g(t, s): t \in I\right\} .
$$

Now, let

$$
y(t)=\int_{0}^{t} f_{2}\left(s, x(\varphi(s)) d_{s} g(t, s), \quad t \in I .\right.
$$

Then the nonlinear functional integral equation (3.1) can be written in the form

$$
x(t)=p(t)+f_{1}(t, y(t)), \quad t \in I .
$$

Hence, the functional integral equation (3.1) is equivalent to the coupled system (3.2) and (3.3).

Now, we study the existence of a continuous solution of the functional integral equation (3.1), which is a solution of the functional integral inclusion (1.1), by getting the continuous solution of the coupled system (3.2) and (3.3).

Definition 3.4. By a solution of the coupled system (3.3) and (3.2) we mean the functions $x, y \in C[0, T]$ satisfying (3.3) and (3.2).

Remark 3.5. From the Lipschitz condition of $\mathrm{f}_{1}$, we have:

$$
\left|f_{1}(t, x)\right|-\left|f_{1}(t, 0)\right| \leqslant\left|f_{1}(t, x)-f_{1}(t, 0)\right| \leqslant k|x|,
$$

i.e.,

$$
\left|f_{1}(t, x)\right| \leqslant k|x|+\sup _{t \in[0, T]}\left|f_{1}(t, 0)\right| \leqslant k|x|+f_{1}^{*}
$$

where

$$
f_{1}^{*}=\sup _{t \in[0, T]}\left|f_{1}(t, 0)\right| .
$$

Let $X$ be the class of all ordered pair $u=(x, y), x, y \in C(I)$ and define the Banach space $X=C(I) \times C(I)$ with the norm

$$
\|(x, y)\|_{x}=\|x\|_{c}+\|y\|_{c} .
$$

Now for the existence of at least one solution, $u=(x, y), x, y \in C(I)$ of the coupled system (3.3), (3.2) we 
have the following theorem.

Theorem 3.6. Let the assumptions (i)-(viii) be satisfied. Then there exists at least one continuous solution $\mathrm{u}=$ $(x, y), x, y \in C(I)$ of the coupled system (3.3), (3.2).

Proof. Let the set $\mathrm{Q}_{\mathrm{r}}$ be defined as

$$
\mathrm{Q}_{\mathrm{r}}=\left\{u=(x, y):(x, y) \in X,\|x\| \leqslant r_{1},\|y\| \leqslant r_{2},\|u\| \leqslant r=r_{1}+r_{2}\right\},
$$

where, $r=\frac{p^{*}+f_{1}^{*}}{1-k}+\frac{a k}{1-b K}$, it is clear that the set $Q_{r}$ is nonempty, bounded, closed and convex. Let $A$ be an operator defined by

$$
\begin{aligned}
A u(t) & =A(x, y)(t)=\left(A_{1} y(t), A_{2} x(t)\right) \\
A_{1} y(t) & =p(t)+f_{1}(t, y(t)), \quad t \in I,
\end{aligned}
$$

and

$$
A_{2} x(t)=\int_{0}^{t} f_{2}\left(s, x(\varphi(s)) d_{s} g(t, s), \quad t \in I,\right.
$$

where for $u=(x, y) \in Q_{r}$, and from Remark (3.5) we have

$$
\left|A_{1} y(t)\right|=\left|p(t)+f_{1}(t, y(t))\right| \leqslant|p(t)|+\left|f_{1}(t, y(t))\right| \leqslant p^{*}+k|x|+f_{1}^{*}=r_{1},
$$

then

$$
\left\|A_{1} y\right\| \leqslant p^{*}+k r_{1}+f_{1}^{*}=r_{1}, \quad r_{1}=\frac{p^{*}+f_{1}^{*}}{1-k} .
$$

Also

$$
\begin{aligned}
\left|A_{2} x(t)\right| & =\left|\int_{0}^{t} f_{2}(s, x(\varphi(s))) d_{s} g(t, s)\right| \\
& \leqslant \int_{0}^{t}\left|f_{2}(s, x(\varphi(s)))\right|\left|d_{s} g(t, s)\right| \\
& \leqslant \int_{0}^{t}[a+b|x(\varphi(s))|] d_{s}\left(\bigvee_{p=0}^{s} g(t, p)\right) \\
\left\|A_{2} x\right\| & \leqslant \int_{0}^{t} a d_{s}\left(\bigvee_{p=0}^{s} g(t, p)\right)+\int_{0}^{t} b|x(\varphi(s))| d_{s}\left(\bigvee_{p=0}^{s} g(t, p)\right) \\
& \leqslant a \int_{0}^{t} d_{s}\left(\bigvee_{p=0}^{s} g(t, p)\right)+b r_{2} \int_{0}^{t} d_{s}\left(\bigvee_{p=0}^{s} g(t, p)\right) \\
& \leqslant\left(a+b r_{2}\right)\left(\bigvee_{s=0}^{t} g(t, s)\right) \\
& \leqslant\left(a+b r_{2}\right) \sup _{t \in I}\left(\bigvee_{s=0}^{t} g(t, s)\right) \\
\left\|A_{2} x\right\| & \leqslant\left(a+b r_{2}\right) K=r_{2}, r_{2}=\frac{a K}{1-b K} .
\end{aligned}
$$

Now

$$
\|A u\|_{X}=\left\|A_{1} y\right\|_{c}+\left\|A_{2} x\right\|_{c} \leqslant r_{1}+r_{2} \leqslant \frac{p^{*}+f_{1}^{*}}{1-k}+\frac{a K}{1-b K}=r .
$$


Then $A Q_{r} \subset Q_{r}$ and the class $\{A u\}, u \in Q_{r}$ is uniformly bounded.

Now, for $u=(x, y) \in Q_{r}$, for all $\epsilon>0, \delta>0$ and for each $t_{1}, t_{2} \in I, t_{1}<t_{2}$ such that $\left|t_{2}-t_{1}\right|<\delta$, we have

$$
\begin{aligned}
\left|A_{1} y\left(t_{2}\right)-A_{1} y\left(t_{1}\right)\right| & =\left|p\left(t_{2}\right)+f_{1}\left(t_{2}, y\left(t_{2}\right)\right)-p\left(t_{1}\right)-f_{1}\left(t_{1}, y\left(t_{1}\right)\right)\right| \\
& \leqslant\left|p\left(t_{2}\right)-p\left(t_{1}\right)\right|+\left|f_{1}\left(t_{2}, y\left(t_{2}\right)\right)-f_{1}\left(t_{1}, y\left(t_{1}\right)\right)\right| \\
& \leqslant\left|p\left(t_{2}\right)-p\left(t_{1}\right)\right|+\left|f_{1}\left(t_{2}, y\left(t_{2}\right)\right)-f_{1}\left(t_{1}, y\left(t_{2}\right)\right)\right|\left|f_{1}\left(t_{1}, y\left(t_{2}\right)\right)-f_{1}\left(t_{1}, y\left(t_{1}\right)\right)\right| \\
& \leqslant\left|p\left(t_{2}\right)-p\left(t_{1}\right)\right|+k\left|t_{2}-t_{1}\right|+k\left|y\left(t_{2}\right)-y\left(t_{1}\right)\right| \\
& \leqslant\left|p\left(t_{2}\right)-p\left(t_{1}\right)\right|+k \delta+k\left|y\left(t_{2}\right)-y\left(t_{1}\right)\right|
\end{aligned}
$$

and

$$
\begin{aligned}
\left|A_{2} x\left(t_{2}\right)-A_{2} x\left(t_{1}\right)\right| \leqslant & \left|\int_{0}^{t_{2}} f_{2}(s, x(\varphi(s))) d_{s} g\left(t_{2}, s\right)-\int_{0}^{t_{1}} f_{2}(s, x(\varphi(s))) d_{s} g\left(t_{1}, s\right)\right| \\
\leqslant & \left|\int_{0}^{t_{2}} f_{2}(s, x(\varphi(s))) d_{s} g\left(t_{2}, s\right)-\int_{0}^{t_{1}} f_{2}(s, x(\varphi(s))) d_{s} g\left(t_{2}, s\right)\right| \\
& +\left|\int_{0}^{t_{1}} f_{2}(s, x(\varphi(s))) d_{s} g\left(t_{2}, s\right)-\int_{0}^{t_{1}} f_{2}(s, x(\varphi(s))) d_{s} g\left(t_{1}, s\right)\right| \\
\leqslant & \left|\int_{t_{1}}^{t_{2}} f_{2}(s, x(\varphi(s))) d_{s} g\left(t_{2}, s\right)\right|+\left|\int_{0}^{t_{1}} f_{2}(s, x(\varphi(s)))\left[d_{s} g\left(t_{2}, s\right)-d_{s} g\left(t_{1}, s\right)\right]\right| \\
\leqslant & \int_{t_{1}}^{t_{2}}[a+b|x(\varphi(s))|] d_{s}\left(\bigvee_{p=0}^{s} g\left(t_{2}, p\right)\right)+\int_{0}^{t_{1}}[a+b|x(\varphi(s))|] d_{s}\left(\bigvee_{p=0}^{s}\left[g\left(t_{2}, p\right)-g\left(t_{1}, p\right)\right]\right) \\
\leqslant & \int_{t_{1}}^{t_{2}}(a+b r) d_{s}\left(\bigvee_{p=0}^{s} g\left(t_{2}, p\right)\right)+\int_{0}^{t_{1}}(a+b r) d_{s}\left(\bigvee_{p=0}\left[g\left(t_{2}, p\right)-g\left(t_{1}, p\right)\right]\right) \\
\leqslant & (a+b r)\left[\int_{t_{1}}^{t_{2}} d_{s}\left(\bigvee_{p=0} g\left(t_{2}, p\right)\right)+\int_{0}^{t_{1}} d_{s}\left(\bigvee_{p=0}^{s}\left(g\left(t_{2}, p\right)-g\left(t_{1}, p\right)\right)\right)\right] \\
\leqslant & (a+b r)\left[\left(\bigvee_{s=0}^{t_{2}} g\left(t_{2}, s\right)-\bigvee_{s=0}^{t_{1}} g\left(t_{2}, s\right)\right)+\bigvee_{s=0}^{t_{1}}\left(g\left(t_{2}, s\right)-g\left(t_{1}, s\right)\right)\right] \\
\leqslant & (a+b r)\left[\bigvee_{s=t_{1}}^{t_{2}} g\left(t_{2}, s\right)+N_{\epsilon}\right],
\end{aligned}
$$

where

$$
N_{\epsilon}=\sup \left\{\bigvee_{s=0}^{t_{2}}\left(g\left(t_{2}, s\right)-g\left(t_{1}, s\right)\right): t_{1}, t_{2} \in I t_{1}<t_{2} ; t_{1}-t_{2} \leqslant \epsilon\right\} .
$$

For the operator $A$ and $u \in Q_{r}$ we have

$$
\begin{aligned}
A u\left(t_{2}\right)-A u\left(t_{1}\right) & =A(x, y)\left(t_{2}\right)-A(x, y)\left(t_{1}\right) \\
& =\left(A_{1} y\left(t_{2}\right), A_{2} x\left(t_{2}\right)\right)-\left(A_{1} y\left(t_{1}\right), A_{2} x\left(t_{1}\right)\right) \\
& =\left(A_{1} y\left(t_{2}\right)-A_{1} y\left(t_{1}\right), A_{2} x\left(t_{2}\right)-A_{2} x\left(t_{1}\right)\right),
\end{aligned}
$$

then

$$
\begin{aligned}
\left\|A u\left(t_{2}\right)-A u\left(t_{1}\right)\right\|_{x} & =\left\|\left(A_{1} y\left(t_{2}\right)-A_{1} y\left(t_{2}\right), A_{2} x\left(t_{2}\right)-A_{2} x\left(t_{1}\right)\right)\right\|_{x} \\
& =\left\|A_{1} y\left(t_{2}\right)-A_{1} y\left(t_{2}\right)\right\|_{C}+\left\|A_{2} x\left(t_{2}\right)-A_{2} x\left(t_{1}\right)\right\|_{C}
\end{aligned}
$$




$$
=\left\|p\left(t_{2}\right)-p\left(t_{1}\right)\right\|+k \delta+k\left\|y\left(t_{2}\right)-y\left(t_{1}\right)\right\|+(a+b r)\left[\bigvee_{s=t_{1}}^{t_{2}} g\left(t_{2}, s\right)+N_{\epsilon}\right] .
$$

This means that the class of functions $\{A u\}$ is equi-continuous on $Q r$. Then by the Arzela-Ascoli Theorem [11], the operator $A$ is compact. It remains to prove the continuity of $A: \operatorname{Qr} \rightarrow \operatorname{Qr}$. Let $u_{n}=\left(x_{n}, y_{n}\right)$ be a sequence in Qr with $x_{n} \rightarrow x$, and $y_{n} \rightarrow y$ and since $f_{2}(t, x(t))$ is continuous in $C(I) \times R$. Then $f_{2}\left(t, x_{n}(t)\right)$ converges to $f_{2}(t, x(t))$, thus $f_{2}\left(t, x_{n}(\varphi(t))\right)$ converges to $f_{2}(t, x(\varphi(t)))$ (see assumption (ii)). Using assumptions (iii) and applying Lebesgue dominated convergence theorem, we get

$$
\lim _{n \rightarrow \infty} \int_{0}^{t} f_{2}\left(s, x_{n}(\varphi(s))\right) d_{s} g(t, s)=\int_{0}^{t} f_{2}(s, x(\varphi(s))) d_{s} g(t, s),
$$

then

$$
\begin{aligned}
& \lim _{n \rightarrow \infty} A_{1} y_{n}(t)=p(t)+\lim _{n \rightarrow \infty} f_{1}\left(t, y_{n}(t)\right)=p(t)+f_{1}(t, y(t)) \\
& \lim _{n \rightarrow \infty} A_{2} x_{n}(t)=\int_{0}^{t} \lim _{n \rightarrow \infty} f_{2}\left(s, x_{n}(\varphi(s))\right) d_{s} g(t, s)=\int_{0}^{t} f_{2}(s, x(\varphi(s))) d_{s} g(t, s)=A_{2} x(t), \\
& \lim _{n \rightarrow \infty} A u_{n}(t)=\lim _{n \rightarrow \infty}\left(A_{1} y_{n}(t), A_{2} x_{n}(t)\right)=\left(\lim _{n \rightarrow \infty} A_{1} y_{n}(t), \lim _{n \rightarrow \infty} A_{2} x_{n}(t)\right)=\left(A_{1} y(t), A_{2} x(t)\right)=A u(t) .
\end{aligned}
$$

Since all conditions of the Schauder fixed-point theorem [28] hold then $A$ has a fixed point $u \in Q_{r}$, and then the system (3.3), (3.2) has at least one continuous solutions $u=(x, y) \in Q_{r}, x, y \in C$ (I). Consequently, the functional integral equation (3.1) has at least one solution $x \in C(I)$.

\section{Asymptotic stability of Volterra-Stieltjes integral inclusion}

We shall show that the solutions of the nonlinear Volterra-Stieltjes integral inclusion are asymptotically stable. The concept of the asymptotic stability of a solution of (1.1) can be given by (as in Banaśa and Rzepka [5]).

Definition 4.1. The solution $x$ of (3.1) is said to be an asymptotically stable solution if for any $\epsilon>0$ there exists $\hat{\top} \in[0, T], \quad T<\infty, \quad \hat{T}=\hat{T}(\epsilon)>0$ such that, if $t \geqslant \hat{T}$ and for every other solutions $y$ of (3.3), (3.2), then

$$
|x(t)-y(t)| \leqslant \epsilon .
$$

Let $x_{1}(t)$ and $x_{2}(t)$ be two solutions of (3.1). Then

$$
\begin{aligned}
\left|y_{1}(t)-y_{2}(t)\right| & \leqslant 1 \int_{0}^{t} f_{2}\left(s, x_{1}(\varphi(s))\right) d_{s} g(t, s)-\int_{0}^{t} f_{2}\left(s, x_{2}(\varphi(s))\right) d_{s} g(t, s) \mid \\
& \leqslant \int_{0}^{t}\left|f_{2}\left(s, x_{1}(\varphi(s))\right)\right|+\left|f_{2}\left(s, x_{2}(\varphi(s))\right)\right|\left|d_{s} g(t, s)\right| \\
& \leqslant \int_{0}^{t} a+b\left|x_{1}(\varphi(s))\right|+a+b\left|x_{2}(\varphi(s))\right| d_{s}\left(\bigvee_{p=0}^{s} g(t, p)\right) \\
& \leqslant \int_{0}^{t}(2 a+2 b) r_{2} d_{s}\left(\bigvee_{p=0}^{s} g(t, p)\right) \\
& \leqslant\left(2 a+2 b r_{2}\right) \bigvee_{s=0}^{t} g(t, s) \leqslant\left(2 a+2 b r_{2}\right) \sup _{t \in I} \bigvee_{s=0}^{t} g(t, s) \leqslant\left(2 a+2 b r_{2}\right) K
\end{aligned}
$$

Also

$$
\left\|x_{1}-x_{2}\right\| \leqslant \sup \left|f_{1}\left(t, y_{1}(t)\right)-f_{1}\left(t, y_{2}(t)\right)\right| \leqslant k\left\|y_{1}-y_{2}\right\| \leqslant k K\left(2 a+2 b r_{2}\right) .
$$

Then, we have proved the following theorem. 
Theorem 4.2. Let the assumptions of Theorem 3.6 be satisfied. If $\mathrm{kK}\left(2 \mathrm{a}+2 \mathrm{br}_{2}\right) \leqslant \epsilon, \mathrm{t} \geqslant \hat{\mathrm{T}}$, then the solution $\mathrm{x}$ of the functional integral inclusion (1.1) is asymptotically stable.

\section{Existence of unique solution}

In this section, we study the uniqueness of the solutions $x \in \mathrm{C}(\mathrm{I})$ of the functional integral inclusion (1.1).

Let us assume the following assumption:

(iv*) let $f_{2}: I \times R \rightarrow R$ be a continuous function satisfies Lipschitz condition for the second variable; that is, there exists a constant $b$ such that

$$
\left\|f_{2}(t, x)-f_{2}(t, y)\right\| \leqslant b\|x-y\|
$$

where $a=\sup _{t \in I}\left|f_{2}(t, 0)\right|$.

Theorem 5.1. Consider the assumptions of Theorem 3.6 be satisfied with replacing condition (iv) by (iv*). If $(1-\mathrm{bkK})<1$, then the functional integral inclusion (1.1) has a unique solution $x \in \mathrm{C}(\mathrm{I})$.

Proof. Let $x_{1}(t)$ and $x_{2}(t)$ be two solutions of the functional integral equation (3.1). Then

$$
\left|x_{1}(t)-x_{2}(t)\right| \leqslant\left|f_{1}\left(t, \int_{0}^{t} f_{2}\left(s, x_{1}(\varphi(s))\right) d_{s} g(t, s)\right)-f_{1}\left(t, \int_{0}^{t} f_{2}\left(s, x_{2}(\varphi(s))\right) d_{s} g(t, s)\right)\right| .
$$

Using Lipschitz condition for $f_{1}$, we obtain

$$
\begin{aligned}
\left|x_{1}(t)-x_{2}(t)\right| & \leqslant k\left|\int_{0}^{t} f_{2}\left(s, x_{1}(\varphi(s)) d_{s} g(t, s)\right)-\int_{0}^{t} f_{2}\left(s, x_{2}(\varphi(s)) d_{s} g(t, s)\right)\right| \\
& \leqslant k \int_{0}^{t}\left|f_{2}\left(s, x_{1}(\varphi(s))\right)-f_{2}\left(s, x_{2}(\varphi(s))\right) \| d_{s} g(t, s)\right| .
\end{aligned}
$$

Using Lipschitz condition for $f_{2}$, we obtain

$$
\begin{aligned}
\left|x_{1}(t)-x_{2}(t)\right| & \leqslant k b \int_{0}^{t}\left|x_{1}(\varphi(s))-x_{2}(\varphi(s))\right| d_{s}\left(\bigvee_{p=0}^{s} g(t, p)\right) \\
& \leqslant b k\left\|x_{1}-x_{2}\right\| \int_{0}^{t} d_{s}\left(\bigvee_{p=0}^{s} g(t, p)\right) \leqslant b k\left\|x_{1}-x_{2}\right\| \bigvee_{s=0}^{t} g(t, s) \leqslant b k\left\|x_{1}-x_{2}\right\| \sup _{t \in I} \bigvee_{s=0}^{t} g(t, s) \\
\left\|x_{1}-x_{2}\right\| & \leqslant b k K\left\|x_{1}-x_{2}\right\| .
\end{aligned}
$$

Then

$$
(1-\mathrm{bkK})\left\|x_{1}-x_{2}\right\|<0 .
$$

This proves the uniqueness of the solution of functional integral equation (3.1).

\subsection{Continuous dependence of the solution}

In this section we are going to study the continues dependence of the unique solution $x \in \mathrm{C}[0, \mathrm{~T}]$ of the functional integral the inclusion (1.1) on the $S_{F_{1}}$ the set of all Lipschitzian selection of $F_{1}$ and the function g.

Theorem 5.2. Let the assumptions of Theorem 5.1 be satisfied. Then the solution of the inclusion (1.1) depends continuously on the $S_{\mathrm{F}_{1}}$ of all Lipschitzian selection of $\mathrm{F}_{1}$. 
Proof. Let $f_{1}(t, x(t))$ and $f_{1}^{*}(t, x(t))$ be two different Lipschitzian selections of $F_{1}(t, x(t))$ such that

$$
\left|f_{1}(t, x(t))-f_{1}^{*}(t, x(t))\right|<\delta, \quad \delta>0, \quad t \in I .
$$

Then for the two corresponding solutions $x(t)$ and $x^{*}(t)$ of inclusion (1.1) we have

$$
\begin{aligned}
x(t)-x^{*}(t)= & f_{1}\left(t, \int_{0}^{t} f_{2}(s, x(\varphi(s))) d_{s} g(t, s)\right)-f_{1}^{*}\left(t, \int_{0}^{t} f_{2}\left(s, x^{*}(\varphi(s))\right) d_{s} g(t, s)\right) \\
\left|x(t)-x^{*}(t)\right| \leqslant & \left|f_{1}\left(t, \int_{0}^{t} f_{2}(s, x(\varphi(s))) d_{s} g(t, s)\right)-f_{1}^{*}\left(t, \int_{0}^{t} f_{2}\left(s, x^{*}(\varphi(s))\right) d_{s} g(t, s)\right)\right| \\
\leqslant & \left|f_{1}\left(t, \int_{0}^{t} f_{2}(s, x(\varphi(s))) d_{s} g(t, s)\right)-f_{1}\left(t, \int_{0}^{t} f_{2}\left(s, x^{*}(\varphi(s))\right) d_{s} g(t, s)\right)\right| \\
& +\left|f_{1}\left(t, \int_{0}^{t} f_{2}\left(s, x^{*}(\varphi(s))\right) d_{s} g(t, s)\right)-f_{1}^{*}\left(t, \int_{0}^{t} f_{2}\left(s, x^{*}(\varphi(s))\right) d_{s} g(t, s)\right)\right| \\
\leqslant & k\left|\int_{0}^{t} f_{2}(s, x(\varphi(s))) d_{s} g(t, s)-\int_{0}^{t} f_{2}\left(s, x^{*}(\varphi(s))\right) d_{s} g(t, s)\right|+\delta \\
\leqslant & k \int_{0}^{t}\left|f_{2}(s, x(\varphi(s)))-f_{2}\left(s, x^{*}(\varphi(s))\right) \| d_{s} g(t, s)\right|+\delta \\
\leqslant & b k \int_{0}^{t}\left|x(\varphi(s))-x^{*}(\varphi(s)) \| d_{s} g(t, s)\right|+\delta \\
\leqslant & b k\left\|x-x^{*}\right\| \int_{0}^{t} d_{s} \bigvee_{p=0} g(t, p)+\delta \\
\leqslant & b k\left\|x-x^{*}\right\| \bigvee_{s=0}^{t} g(t, s)+\delta \leqslant b k\left\|x-x^{*}\right\| \sup _{t \in I} \bigvee_{s=0}^{t} g(t, s)+\delta \leqslant b k\left\|x-x^{*}\right\| K+\delta \\
\left\|x-x^{*}\right\| \leqslant & (1-b k K)^{-1} \delta=\epsilon .
\end{aligned}
$$

Thus the above inequality gives that

$$
\left\|x-x^{*}\right\| \leqslant \epsilon .
$$

This mean that the solution of the of inclusion (1.1) depends continuously on the set $S_{\mathrm{F}_{1}}$ of all Lipschitzian selection of $F_{1}$. This complete the proof.

Theorem 5.3. Let the assumptions of Theorem 5.1 be satisfied. Then the solution of the inclusion (1.1) depends continuously on the function $\mathrm{g}$.

Proof. Let $g(t, x(t))$ and $g^{*}(t, x(t))$ be two different functions, and let $\delta>0$ be given such that

$$
\left|g(t, x(t))-g^{*}(t, x(t))\right|<\delta, \quad t \in I .
$$

Then for the two corresponding solutions $x(t)$ and $x^{*}(t)$ of inclusion (1.1) we have

$$
\begin{aligned}
x(t)-x^{*}(t) & =f_{1}\left(t, \int_{0}^{t} f_{2}(s, x(\varphi(s))) d_{s} g(t, s)\right)-f\left(t, \int_{0}^{t} f_{2}\left(s, x^{*}(\varphi(s))\right) d_{s} g^{*}(t, s)\right) \\
\left|x(t)-x^{*}(t)\right| & \leqslant\left|f_{1}\left(t, \int_{0}^{t} f_{2}(s, x(\varphi(s))) d_{s} g(t, s)\right)-f\left(t, \int_{0}^{t} f_{2}\left(s, x^{*}(\varphi(s))\right) d_{s} g^{*}(t, s)\right)\right| \\
& \leqslant\left|f_{1}\left(t, \int_{0}^{t} f_{2}(s, x(\varphi(s))) d_{s} g(t, s)\right)-f_{1}\left(t, \int_{0}^{t} f_{2}\left(s, x^{*}(\varphi(s))\right) d_{s} g(t, s)\right)\right|
\end{aligned}
$$




$$
\begin{aligned}
& +\left|f_{1}\left(t, \int_{0}^{t} f_{2}\left(s, x^{*}(\varphi(s))\right) d_{s} g(t, s)\right)-f\left(t, \int_{0}^{t} f_{2}\left(s, x^{*}(\varphi(s))\right) d_{s} g^{*}(t, s)\right)\right| \\
\leqslant & k \int_{0}^{t} \mid f_{2}\left(s, x(\varphi(s))-f_{2}\left(s, x^{*}(\varphi(s)) \mid d_{s} g(t, s)\right)\right. \\
& +k \int_{0}^{t}\left|f_{2}\left(s, x^{*}(\varphi(s))\right)\right| d_{s}\left|g(t, s)-g^{*}(t, s)\right| \\
\leqslant & k \int_{0}^{t}\left|f_{2}(s, x(\varphi(s)))-f_{2}\left(s, x^{*}(\varphi(s))\right)\right|\left|d_{s} g(t, s)\right| \\
& +k \int_{0}^{t}\left(\left|f_{2}\left(t, x^{*}(\varphi(s))\right)-f_{2}(t, 0)\right|-\left|f_{2}(t, 0)\right|\right) d_{s}\left|g(t, s)-g^{*}(t, s)\right| \\
\leqslant & b k \int_{0}^{t}\left|x(\varphi(s))-x^{*}(\varphi(s)) \| d_{s} g(t, s)\right| \\
& +k \int_{0}^{t}\left(a+b\left|x^{*}(\varphi(s))\right|\right) d_{s}\left|g(t, s)-g^{*}(t, s)\right| \\
\leqslant & b k\left\|x-x^{*}\right\| \int_{0}^{t} d_{s} \bigvee_{p=0}^{s} g(t, p)+k(a+b r) \int_{0}^{t} d_{s} \bigvee_{p=0}^{s}\left|g(t, p)-g^{*}(t, p)\right| \\
\leqslant & b k\left\|x-x^{*}\right\| \bigvee_{s=0}^{t} g(t, s)+k(a+b r) \bigvee_{s=0}^{t}\left|g(t, s)-g^{*}(t, s)\right| \\
\leqslant & b k\left\|x-x^{*}\right\| \sup _{t \in I} \bigvee_{s=0}^{t} g(t, p)+k(a+b r)\left|g(t, t)-g^{*}(t, t)-g(t, 0)+g^{*}(t, 0)\right| \\
\leqslant & b k\left\|x-x^{*}\right\| K+k(a+b r)\left|g(t, t)-g^{*}(t, t)-g(t, 0)+g^{*}(t, 0)\right| \\
\leqslant & b k\left\|x-x^{*}\right\| K+k(a+b r)\left[\left|g(t, t)-g^{*}(t, t)\right|+\left|g(t, 0)-g^{*}(t, 0)\right|\right] \\
\leqslant & b k\left\|x-x^{*}\right\| K+2 \delta k(a+b r), \\
\| & 2 \delta k(a+b r)(1-b k K)^{-} 1=\epsilon .
\end{aligned}
$$

Then the above inequality gives that

$$
\left\|x-x^{*}\right\| \leqslant \epsilon .
$$

This mean that the solution of the of inclusion (1.1) depends continuously on the functional g. This completes the proof.

\section{Volterra integral equation of fractional order}

In this section, we will consider the fractional integral inclusion, which has the form

$$
x(t) \in p(t)+F_{1}\left(t, \int_{0}^{t} \frac{(t-s)^{\alpha-1}}{\Gamma(\alpha)} f_{2}(s, x(\varphi(s)) d s)\right), t \in I,
$$

where $t \in I=[0, T]$ and $\alpha \in(0,1)$. Moreover, $\Gamma(\alpha)$ denotes the gamma function. Let us mention that (6.1) represents the so-called nonlinear Volterra integral inclusion of fractional orders. Recently, the inclusion of such a type was intensively investigated in some papers [1, 15]. Now, we show that the functional integral inclusion of fractional orders (6.1) can be treated as a particular case of the set-valued functional integral equation of Volterra-Stieltjes (1.1) studied in Section 3.

Indeed, we can consider the function $g(t, s)=g: \triangle \rightarrow R$ defined by the formula

$$
g(t, s)=\frac{t^{\alpha}-(t-s)^{\alpha}}{\Gamma(\alpha+1)}
$$


Note that the function $g$ satisfies assumptions (v)-(viii) in Theorem 3.6, see [6, 22]. Then, it can be easily seen that (6.1) can be written in the form of (1.1). Now, we can formulate the following existence results concerning the Volterra integral inclusion of fractional order (6.1).

Theorem 6.1. Under the assumptions (i)-(iv) of Theorem 3.6, the fractional integral inclusion (6.1) has at least one continuous solution $x \in \mathrm{C}(\mathrm{I})$.

Theorem 6.2. Under the assumptions of Theorem 5.1, the fractional integral inclusion (6.1) has exactly one unique solution $x \in \mathrm{C}(\mathrm{I})$.

\subsection{Differential inclusion}

Consider now the initial value problem of the differential inclusion (1.2) with the initial data (1.3).

Theorem 6.3. Let the assumptions of Theorem 6.1 be satisfied. Then the initial value problem (1.2)-(1.3) has at least one solution $\mathrm{x} \in \mathrm{C}(\mathrm{I})$.

Proof. Let $y(t)=\frac{d x(t)}{d t}$. Then the inclusion (1.2), will be

$$
y(t) \in F_{1}\left(t, I^{1-\beta} y(t)\right) .
$$

Letting $f_{2}(t, x)=x(t), \varphi(t)=t$, and $\alpha=1-\beta$ applying Theorem 6.1 on the functional inclusion (6.2), we deduce that there exists at least one continuous solution $y \in C(I)$ of the functional inclusion (6.2). This implies that the existence of solution $x \in \mathrm{C}(\mathrm{I})$,

$$
x(t)=x_{\circ}+\int_{0}^{t} y(s) d s
$$

of the initial-value problem (1.2)-(1.3).

\section{Further discussions}

This section is devoted to discussing some special cases of the inclusion (1.1). First of all let us notice that the classical nonlinear Volterra integral inclusion

$$
x(t) \in p(t)+F_{1}\left(t, \int_{0}^{t} f_{2}(s, x(\varphi(s)) d s)\right.
$$

is a special case of inclusion (1.1) if we put $g(t, s)=s$. Indeed, assumptions (v)-(viii) are trivially satisfied for $\mathrm{g}$. This implies that inclusion (7.1) can be investigated with help of Theorem 3.6 to observe that assumptions of that theorem are automatically satisfied for inclusion (7.1).

Next, let us consider the integral inclusion of Volterra type having the form

$$
x(t) \in p(t)+F_{1}\left(t, \int_{0}^{t} \frac{t}{t+s} f_{2}(s, x(\varphi(s))) d s\right),
$$

which represents the Chandrasekhar's integral inclusion.

Now we show that (7.2) is a special case of inclusion (1.1), to do this let us consider the function $g: \triangle \rightarrow R$, defined by the formula

$$
g(t, s)= \begin{cases}t \ln \frac{t+s}{t}, & \text { for } t>0 \text { and } s \geqslant 0 \\ 0, & \text { for } t=0 \text { and } s \geqslant 0\end{cases}
$$

Using standard methods of mathematical analysis it can be seen that the function $g(t, s)$ defined by $(7.3)$ satisfies assumptions (v)-(viii) of Theorem 3.6. Then we see that inclusion (1.1) can be written in the form (7.2). 
Moreover, let us remember that if the function $g(t, s)$ satisfies assumptions (v)-(viii) of Theorem 3.6, then it represents the distribution function of a two dimensional random variable $[7,8]$. The converse implication is also "almost" valid $[8,14]$. In particular, we may consider the function to be the distribution function of the continuous type two-dimensional random variable. Such a function has the form

$$
g(t, s)=\int_{0}^{t}\left(\int_{0}^{s} p(z, y) d y\right) d z
$$

where $p(z, y)$ is the so-called density function [7]. Obviously this function satisfies assumptions (v)-(viii). Moreover, we have

$$
d_{s} g(t, s)=\int_{0}^{t} p(s, y) d y,
$$

and in this case integral inclusion of Volterra-Stiltjes type (1.1) has the form

$$
x(t) \in p(t)+F_{1}\left(t, \int_{0}^{t} f_{2}\left(s, x(\varphi(s))\left(\int_{0}^{t} p_{1}(s, y) d y\right) d s\right),\right.
$$

let us mention that this inclusion is the usual nonlinear Volterra integral equation. Thus, based on Theorem 3.6 we deduce that (1.1) can be written in the form (7.4).

\section{Acknowledgment}

The authors wish to express their gratitude to the anonymous referees for their valuable comments and suggestions, which allowed to improve an early version of this work.

\section{References}

[1] S. Al-Issa, A. M. A. El-Sayed, Positive integrable solutions for nonlinear integral and differential inclusions of fractionalorders, Comment. Math., 49 (2009), 171-177. 6

[2] J. Appell, J. Banaś, N. Merentes, Bounded variation and around, De Gruyter, Berlin, (2014). 2

[3] J. P Aubin, A. Cellina, Differential inclusions: set-valued maps and viability theory, Springer-Verlag, Berlin, (2012). 2.2

[4] J. Banaś, A. Dubiel, Solutions of a quadratic Volterra-Stieltjes integral equation in the class of functions converging at infinity, Electron. J. Qual. Theory Differ. Equ., 2018 (2018), 17 pages. 1

[5] J. Banaś, B. Rzepka, On existence and asymptotic stability of solutions of a nonlinear integral equation, J. Math. Anal. Appl., 284 (2003), 165-173. 4

[6] J. Banaś, T. Zajac, A new approach to the theory of functional integral equations of fractional order, J. Math. Anal. Appl., 375 (2011), 375-387. 1, 3.1, 3.2, 3.3, 6

[7] P. Billingsley, Probability and Measure, Wiley, New York, (1979). 7

[8] A. M. Bruckner, Differentiation of real functions, Springer, Berlin, (2006). 7

[9] A. Cellina, S. Solimini, Continuous extensions of selections, Bull. Polish Acad. Sci. Math., 35 (1989), 573-581. 2.1

[10] K.-S. Chiu, T. X. Li, Oscillatory and Periodic Solutions of Differential Equations with Piecewise Constant Generalized Mixed Arguments, Math. Nachr., 2019 (2019), 12 pages. 1

[11] R. F. Curtain, A. J. Pritchard, Functional Analysis in Modern Applied Mathematics, Academic press, London-New York, (1977). 3

[12] M. A. Darwish, J. Banaś, Existence and characterization of solutions of nonlinear Volterra-Stieltjes integral equations in two variables, Abstr. Appl. Anal., 2014 (2014), 11 pages. 1

[13] M. A. Darwish, J. Henderson, Nondecreasing solutions of a quadratic integral equation of Urysohn-Stieltjes type, Rocky Mountain J. Math., 42 (2012), 545-566. 1

[14] K. Deimling, Nonlinear Functional Analysis, Springer-Verlag, Berlin, (1985). 7

[15] A. M. A. El-Sayed, S. M. Al-Issa, Global Integrable Solution for a Nonlinear Functional Integral Inclusion, SRX Math., 2010 (2010), 4 pages. 1, 6

[16] A. M. A. El-Sayed, S. M. Al-Issa, Monotonic continuous solution for a mixed type integral inclusion of fractional order, J. Math. Appl., 33 (2010), 27-34.

[17] A. M. A. El-Sayed, S. M. Al-Issa, Existence of continuous solutions for nonlinear functional differential and integral inclusions, Malaya J. Mat., 7 (2019), 541-544.

[18] A. M. A. El-Sayed, S. M. Al-Issa, Monotonic integrable solution for a mixed type integral and differential inclusion of fractional orders, Int. J. Differ. Equations Appl., 18 (2019), 10 pages. 1 
[19] A. M. A. El-Sayed, S. M. Al-Issa, Monotonic solutions for a quadratic integral equation of fractional order, AIMS Mathematics, 4 (2019), 821-830. 1

[20] A. M. A. El-Sayed, S. M. Al-Issa, Existence of integrable solutions for integro-differential inclusions of fractional order; coupled system approach, J. Nonlinear Sci. Appl., 13 (2020), 180-186. 1

[21] A. M. A. El-Sayed, S. M. Al-Issa, On the existence of solutions of a set-valued functional integral equation of Volterra-Stieltjes type and some applications, Adv Difference Equ., 2020 (2020), 16 pages. 1

[22] A. M. A. El-Sayed, W. G. El-Sayed, A. A. H. Abd El-Mowla, Weak solutions of fractional order differential equations via Volterra-Stieltjes integral operator, J. Math. Appl., 40 (2017), 85-96. 2, 6

[23] A. M. A. El-Sayed, A.-G. Ibrahim, Multivalued fractional differential equations, Appl. Math. Comput., 68 (1995), 15-25. 1, 2.2

[24] A. M. A. El-Sayed, A.-G. Ibrahim, Set-valued integral equations of fractional-orders, Appl. Math. Comput., 118 (2001), 113-121. 1, 2.3

[25] A. Fyszkowski, Continuous selection for a class of non-convex multivalued maps, Studia Math., 76 (1983), 163-174. 2.1

[26] A.-G. Ibrahim, A. M. A. El-Sayed, Definite integral of fractional order for set-valued function, J. Fract. Calc., 11 (1997), 81-87. 1

[27] K. Kuratowski, C. Ryll-Nardzewski, Ageneral theorem on selectors, Bull. Acad. Polon. Sci. Sér. Sci. Math. Astronom. Phys., 13 (1965), 397-403. 1, 2.1, 2

[28] V. Lakshmikantham, S. Leela, Differential and Integral Inequalities, Academic press, New York-London, (1969). 3

[29] K. S. Miller, B. Ross, An Introduction to the fractional calculus and fractional differential equations, John Wiley \& Sons, New York, (1993). 1

[30] I. Podlubny, Fractional Differential Equations, Academic Press, San Diego, (1999). 1 